Provided for non-commercial research and educational use only. Not for reproduction or distribution or commercial use.

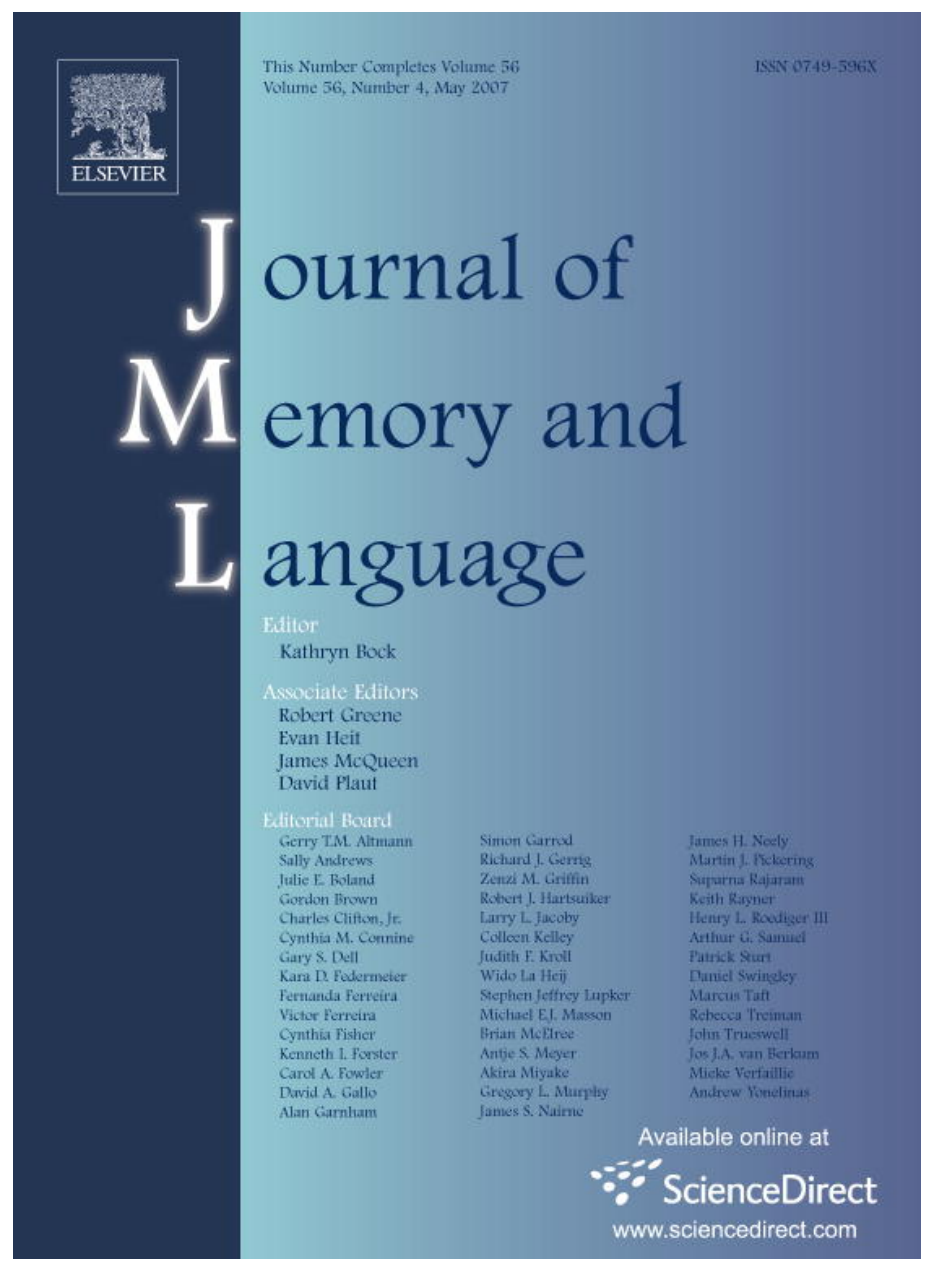

This article was originally published in a journal published by Elsevier, and the attached copy is provided by Elsevier for the author's benefit and for the benefit of the author's institution, for non-commercial research and educational use including without limitation use in instruction at your institution, sending it to specific colleagues that you know, and providing a copy to your institution's administrator.

All other uses, reproduction and distribution, including without limitation commercial reprints, selling or licensing copies or access,

or posting on open internet sites, your personal or institution's website or repository, are prohibited. For exceptions, permission may be sought for such use through Elsevier's permissions site at: 


\title{
The contribution of relatedness and distinctiveness to emotionally-enhanced memory
}

\author{
Deborah Talmi *, Betty T.C. Luk, Lucy M. McGarry, Morris Moscovitch * \\ Department of Psychology, University of Toronto, 100 St. George Street, Toronto, Ont., Canada M5S $3 G 3$
}

Received 4 December 2005; revision received 30 December 2006

Available online 13 March 2007

\begin{abstract}
Emotional events are more organized and distinctive than neutral events. We asked whether organization and distinctiveness can account for emotionally-enhanced memory. To examine organization, we compared memory for arousing, negatively-valenced pictures, and inter-related neutral pictures. To examine distinctiveness, we manipulated list composition, and compared mixed lists, which contained emotional and neutral items, to pure lists, which contained only items of a single type and removed the relative-distinctiveness advantage of emotional items. We show that emotional memory is enhanced in immediate memory tests as long as either organization or distinctiveness is allowed to play a role. When these effects are removed, in the comparison of emotional and related neutral items in pure lists, the emotional memory advantage is eliminated. Examining the contribution of mediating cognitive factors at a behavioral and neural level is crucial if we are to understand how emotion influences memory.
\end{abstract}

(c) 2007 Elsevier Inc. All rights reserved.

Keywords: Memory; Emotion; Arousal; Relatedness; Distinctiveness; Free recall

The mnemonic advantage of emotional events has been replicated frequently in the laboratory (for review, see Dolan, 2002; Labar \& Cabeza, 2006), but the mechanism(s) underlying it are not understood fully. LaBar and Cabeza review evidence that emotional arousal can modulate the long-term consolidation of memory traces, so that when tested hours and days after study, memory for emotional items is better than memory for neutral ones. This neurobiological model can account for the prolonged effects emotion has on memory, but not for the mnemonic benefits that are already seen in immediate memory tests, before differential consolida-

\footnotetext{
* Corresponding authors. Fax: +1 4169784811.

E-mail addresses: debbie@psych.utoronto.ca (D. Talmi), momos@psych.utoronto.ca (M. Moscovitch).
}

tion had a chance to exert its effects (e.g. Hamann, Cahill, McGaugh, \& Squire, 1997; Kensinger, Brierley, Medford, Growdon, \& Corkin, 2002; LaBar \& Phelps, 1998; MacKay et al., 2004; Phelps, LaBar, \& Spencer, 1997; Phelps et al., 1998). The present study examines the role of cognitive variables as mediating factors of the emotional memory advantage - the possibility that emotionality is crucial, but is not the direct cause, of the better memory for emotional items. Specifically, we propose that emotion may have important memory consequences because it links separate events together, and also allows them to stand out relative to background neutral events. Since both organization and distinctiveness can enhance memory (Hunt \& McDaniel, 1993), regardless of emotionality, the effect of emotion on these factors could account for the advantage of emotional items over neutral ones. While mediation explanations 
could account for both the immediate and the delayed memory advantage of emotional items, we focus on immediate tests here to remove the complicating factor of differential consolidation that is present in delayed testing situations.

\section{Emotion and organization}

Emotional items are related because they share category membership and a thematic relationship (the words "torture" and "pain", a picture of a gun and a picture of a dead body). Recently, we have shown that enhanced memory for emotional words, which was present in the comparison with randomly-selected neutral words, was eliminated in the comparison with equally related neutral words (Talmi \& Moscovitch, 2004). Since relatedness itself can enhance memory by improving list organization at encoding as well as aiding retrieval (Gardiner, Craik, \& Birtwistle, 1972; Hunt \& McDaniel, 1993; Mandler, 1967), the finding that emotional memory advantage was eliminated when relatedness was controlled supported the hypothesis that differential relatedness can completely account for emotionallyenhanced memory for words (for similar suggestions see Maratos \& Rugg, 2001; Phelps et al., 1997).

Because the emotional words Talmi and Moscovitch (2004) used were not taboo words, they may not have been arousing enough to allow the emotional memory advantage to be manifested above and beyond the effects of differential relatedness. Buchanan, Etzel, Adolphs, and Tranel (2006) replicated the finding of equivalent memory for emotional and related-neutral words, but extended it to show that taboo words, which were more arousing than non-taboo emotional words, were recalled better than related-neutral words (see also MacKay et al., 2004). Since emotional arousal, rather than valence, is believed to be responsible for the memory enhancement effects of emotional material (Hamann, 2001; Zald, 2003), it is important to ensure that the emotional material used to study this effect is as arousing as possible. Thus, our first goal in the present study was to find out whether equating relatedness eliminates the emotional advantage even when emotional items are very arousing. Here we used negatively-valenced, arousing photographs depicting scenes of violence and mutilation as the emotional stimuli. The comparison neutral stimuli consisted of randomly-selected neutral pictures, as well as neutral pictures of domestic scenes which were as related to one another as the emotional pictures.

\section{Emotion and distinctiveness}

Emotional items are not only organized better than neutral items, but also are more distinct in both an 'absolute' and a 'relative' sense. Schmidt (1991; see also Hunt \& McDaniel 1993) defines absolute ('secondary') distinctiveness as the result of the limited overlap of the features of the item (e.g. an image of a hungry child) with the 'active conceptual framework', a term used to define the typical items that are stored pre-experimentally in long-term memory (e.g. neutral images of people, buildings, and vehicles). Emotional items are more distinct than neutral ones because they possess unique features which are not shared with other items that typically make up the 'active conceptual framework'.

A core feature that emotional items do not typically share with neutral items is their relevance to participants' goals (Lazarus, 1991). The idea is that an image like that of a hungry child has an emotional impact because it is relevant to the observer's goal, such as a goal to care for conspecifics. Goal-relevance, in turn, makes emotional items interesting (Bradley, 1994) and allows them to evoke unique physiological responses (Ochsner, 2000), additional features that emotional items do not share with typical neutral items in the active conceptual framework, and add to their absolute distinctiveness or 'unusualness' (Adolphs, Denburg, \& Tranel, 2001). In this sense, then, goal-relevance influences both the ability of an event to evoke emotions, as well as the distinctiveness of the event.

According to Schmidt (1991), relative ('primary') distinctiveness is a result of an item's limited overlap with the items stored in the active conceptual framework which participants maintain in working memory. For example, a black letter in a stimulus stream is not absolutely distinct, but is relatively distinct when all other letters are blue. Within the typical experimental context, when emotional items are presented alongside neutral ones, their absolute distinctiveness makes them stand out relative to the neutral items, so that they are both absolutely and relatively distinct.

It is possible to change an item's relative distinctiveness by changing participants' active conceptual framework. This can be done by presenting absolutely-distinct items either in a mixed list that contains indistinct items, or in a pure list, that blocks item type. Items with absolute distinctiveness will only be relatively distinct in the mixed-list condition. For example, a picture of a gun is relatively distinct after viewing a series of images from daily life, but less so after viewing a series of robbery images. After reviewing the literature, Schmidt (1991) concluded that only relative, but not absolute, distinctiveness enhances memory. That conclusion was based on findings with bizarre sentences, with humorous sentences, and with orthographically distinct items - all of which are remembered better in mixed lists that contain common, non-humorous and orthographically regular items, but not in pure lists (Einstein \& McDaniel, 1987; Hunt \& Elliot, 1980; Schmidt, 1994). Recently, Dewhurst and Parry (2000) 
have shown that the enhancing effect of emotion on recollection was present only in a mixed list of emotional and random-neutral words, but not when pure lists were compared, and suggested that differential relative distinctiveness underlies the effect of emotion. However, when we controlled relatedness (Talmi \& Moscovitch, 2004) we did not find a benefit of emotional over related-neutral items on recall in either pure or mixed lists of words. We have proposed above that both distinctiveness and emotionality stem from goalrelevance. If picutres are more goal-relevant than words, emotional pictures might not only be more emotionally arousing, but may also be more distinct than emotional words, relative to their respective non-targets. This leaves open the possibility that the emotional words we have used previously were not distinct relative to the neutral words. The second goal of the present study was to determine whether the memory advantage for highly arousing emotional items is eliminated when they are no longer relatively distinct. To accomplish this goal, we manipulated list composition and compared emotionally-enhanced memory in pure-list and mixed-list conditions.

Paradoxically, distinctiveness and organization can work in concert to improve memory (Hunt \& McDaniel, 1993) by endowing items with more inter-item associations as well as intra-item elaborations (Mandler, 1980). For instance, both organization and distinctiveness contribute to the 'self-reference effect', the mnemonic superiority that results from relating items to the self (Symons \& Johnson, 1997). This self-reference memory advantage is thus akin to the advantage emotional items have relative to random-neutral items, which are less distinct and less well-organized. Because relatedness and distinctiveness interact, it is important to control relatedness when examining distinctiveness, and vice versa; this links the two goals of this study. It is worth noting that although memory for non-distinct items suffers from the inclusion of distinct items in the list, importantly, it has been shown previously that the organization of non-distinct items does not change as a result (Schmidt, 1984). For this reason, neutral items should be organized just as well in a pure list of neutral items, or in a mixed list that includes emotional items.

To summarize, the following experiments examined the possibility that equating the distinctiveness and relatedness of emotional and neutral items would eliminate the memory advantage that emotional items have over neutral ones. In the experiments reported here, we presented items in pure or mixed lists, and compared emotionally-arousing items to both equally-related neutral items and randomly selected neutral items. To preview, our findings suggest that the memory advantage of emotional items is determined by relatedness and relative distinctiveness.

\section{Experiment 1}

In Experiment 1, participants studied three pure lists of emotional, random neutral, and related neutral items, or three mixed list that combined all these item types. Memory was tested after a 1-min filled interval. We predicted that regardless of list composition, because of their higher relatedness, emotional items would be recalled better than random neutral items, thereby replicating the classic effect. If negative emotion improves memory in addition to its effect on relatedness, memory for emotional items should also be better than memory for equally related neutral items. If emotionality improves memory in addition to its effects on distinctiveness, then emotional items should be remembered better than related neutral items not only in the mixed list condition (where relative distinctiveness could play a role) but also in the pure list condition (where it cannot). By contrast, if the memory advantage of emotional over neutral items is dependent on their higher relatedness and distinctiveness, it would be eliminated in the comparison of pure lists of emotional and related-neutral items.

Memory was assessed with a free recall task. Free recall is a less typical memory test of pictorial material than is recognition, but it has been successfully employed in previous studies of emotion and memory (Blake, Varnhagen, \& Parent, 2001; Bradley, Greenwald, Petry, \& Lang, 1992; Hamann, Ely, Grafton, \& Kilts, 1999; Kensinger et al., 2002; Palomba, Angrilli, \& Mini, 1997), which all found that emotional pictures were recalled better than neutral ones. The free recall paradigm allowed us to test memory immediately, when recognition memory is at ceiling. It is also more akin to real-life recall of visually experienced scenes, and, in this sense, is more ecologically valid.

\section{Method}

\section{Participants}

Fifty-one undergraduate students from the University of Toronto ( 17 males, mean age $19.67, \mathrm{SD}=2.03$ ) participated in the study for course credit. All participants in the following experiments gave informed consent; to comply with ethical guidelines, the consent form explicitly mentioned that they would be seeing potentially disturbing and graphic pictures. No participant in these experiments reported having a history of neurological or mental illness. Participants were randomly assigned to the two list-composition manipulation conditions, pure list (30 participants) and mixed list (21 participants).

\section{Material}

A set of 15 negative, 15 related neutral (domestic scenes) and 15 random neutral pictures, was selected from the International Affective Picture System (IAPS, Lang, Bradley, \& Cuthbert, 1999) and internet sources. 
The pictures were adjusted in size to $400 \times 500$ pixels. These pictures were selected from a larger picture pool on the basis of an extensive pilot study, in which two groups of 12 participants each (of the same age group and from the same population as the experimental participants) rated 160 pictures on emotional valence, arousal, visual complexity, brightness, and inter-item relatedness. The first group rated all pictures for valence and emotional arousal using the computerized Self Assessment Manikin (SAM, Bradley \& Lang, 1994), and also for the visual complexity and brightness using 7-point Likert scales. Agreement among raters was high, standardized item $\alpha>.90$. The second group rated the pictures for semantic relatedness on a 7-point Likert scale, following the procedure described in Talmi and Moscovitch (2004). Research on semantic relatedness, measured with a similar procedure, has shown that semantic similarity is an important aspect of semantic relatedness and a major organizing principle in semantic memory, as demonstrated in the strong semantic priming among semantically similar words (McRae \& Boisvert, 1998). For the semantic relatedness judgment, participants were asked to consider picture content rather than superficial similarity in colors or layout. They were also given examples for the types of content that can make pictures related; for instance, pictures are related when they depict exemplars from the same category (a hand gun and a rifle; jogging and walking), or thematically linked as script relations (clouds and umbrella). The entire picture pool was divided into four sets of 40 pictures each, and all possible picture pairs in each set (780 ratings per set) were presented and rated in a random order. Again, agreement among raters was high, standardized item $\alpha>90$. Each picture received a relatedness score, computed as the average rated relatedness of that picture with all other pictures of its type in the list to which it was allocated. Picture scores were computed across participants, averaged for each picture and analyzed with separate analyses of variance (ANOVAs).

In the pure list condition, we analyzed picture scores (see Table 1, set 1 ) with a series of univariate ANOVAs. Picture types statistically differed in valence, $F(2,42)=136.515, p<.001$, and in emotional arousal, $F(2,42)=135.944, p<.001$. Planned contrasts showed that the negative pictures were significantly more negatively valenced, $p<.001$, and more arousing, $p<.001$, relative to both types of neutral pictures, but the two neutral types did not differ in valence, $p>.10$, or arousal, $p>.10$. Picture types also differed in semantic relatedness, $F(2,42)=44.89, p<.001$, with lower scores for random neutral pictures, $p<.001$, but no difference between the related neutral categorized and negative pictures, $p>.10$. Picture types did not differ on people presence, $p>.10$, visual complexity, $p>.10$, or brightness, $p>.10$. In the mixed list condition (see Table 2), we ana-
Table 1

Average scores for pictures used in the pure-list conditions

\begin{tabular}{lccc}
\hline & Emotional & $\begin{array}{c}\text { Related- } \\
\text { neutral }\end{array}$ & $\begin{array}{c}\text { Random- } \\
\text { neutral }\end{array}$ \\
\hline Set 1 & & & \\
Arousal & $5.74(0.80)$ & $2.66(0.39)$ & $2.91(0.41)$ \\
Valence & $2.80(0.63)$ & $5.05(0.35)$ & $5.11(0.23)$ \\
Visual complexity & $5.29(0.84)$ & $5.11(0.74)$ & $5.20(1.10)$ \\
Brightness & $4.87(0.72)$ & $5.40(0.94)$ & $4.90(0.65)$ \\
Relatedness & $1.77(0.29)$ & $1.69(0.16)$ & $1.15(0.09)$ \\
People presence & $0.73(0.46)$ & $0.53(0.52)$ & $0.67(0.49)$ \\
Set 2 & & & \\
Arousal & $5.42(0.71)$ & $2.59(0.52)$ & $2.94(0.58)$ \\
Valence & $2.89(0.56)$ & $4.93(0.36)$ & $5.09(0.44)$ \\
Visual complexity & $5.21(1.00)$ & $5.04(0.71)$ & $4.90(1.61)$ \\
Brightness & $5.10(0.88)$ & $5.21(1.02)$ & $5.19(0.94)$ \\
Relatedness & $1.60(0.36)$ & $1.52(0.25)$ & $1.09(0.11)$ \\
People presence & $0.73(0.46)$ & $0.53(0.52)$ & $0.47(0.52)$ \\
\hline
\end{tabular}

Note. Pilot participants rated pictures for arousal and valence using the SAM scale; scores are converted to numbers such that "1" represents "least arousing"/"most negative", and 9 represents "most arousing"/"most positive". They rated pictures for visual complexity, brightness and relatedness on a 1-7 Likert scale with " 1 " representing a low value and 7 representing a high value. Pictures got a score of " 1 " if they depicted people and " 0 " if they did not, and the proportion was calculated. See text for more details. Values in the table represent means, with standard deviation in parenthesis.

stan

Table 2

Average scores for pictures used in the mixed-list conditions

\begin{tabular}{lcccc}
\hline & List & Emotional & $\begin{array}{c}\text { Related- } \\
\text { neutral }\end{array}$ & $\begin{array}{c}\text { Random- } \\
\text { neutral }\end{array}$ \\
\hline Set 1 & & & & \\
Arousal & 1 & $5.64(0.76)$ & $2.79(0.31)$ & $2.90(0.51)$ \\
& 2 & $5.66(0.55)$ & $2.43(0.36)$ & $2.81(0.28)$ \\
Valence & 3 & $5.91(1.15)$ & $2.76(0.47)$ & $3.03(0.46)$ \\
& 1 & $2.91(0.59)$ & $5.33(0.35)$ & $5.03(0.12)$ \\
& 2 & $2.59(0.45)$ & $4.94(0.28)$ & $5.11(0.21)$ \\
Visual complexity & 3 & $2.89(0.88)$ & $4.87(0.26)$ & $5.19(0.33)$ \\
& 1 & $5.40(1.04)$ & $5.47(0.55)$ & $5.34(0.61)$ \\
Brightness & 2 & $5.37(0.77)$ & $4.64(0.64)$ & $4.76(1.65)$ \\
& 3 & $5.10(0.86)$ & $5.23(0.86)$ & $5.50(0.85)$ \\
Relatedness & 1 & $4.90(0.84)$ & $5.93(0.67)$ & $4.77(0.63)$ \\
& 2 & $4.91(0.40)$ & $5.47(1.20)$ & $5.17(0.86)$ \\
& 3 & $4.80(0.98)$ & $4.79(0.59)$ & $4.74(0.46)$ \\
People presence & 1 & $1.77(0.29)$ & $1.79(0.15)$ & $1.15(0.07)$ \\
& 2 & $1.79(0.26)$ & $1.74(0.07)$ & $1.09(0.05)$ \\
& 3 & $1.74(0.37)$ & $1.55(0.13)$ & $1.21(0.10)$ \\
& 2 & $0.80(0.45)$ & $0.60(0.55)$ & $0.80(0.45)$ \\
& 3 & $0.60(0.45)$ & $0.60(0.55)$ & $0.40(0.55)$ \\
& & & & \\
& & & &
\end{tabular}

Note. See note for Table 1. 
lyzed picture scores with a series of 3 (mixed lists) $\times 3$ (picture type) univariate ANOVA analyses. There was no significant main effect of mixed lists or interactions between mixed lists and picture type, all $p$ values $>.10$. Planned contrasts showed that the effect of picture type was the same as in the pure list condition.

In addition to the experimental pictures, we also used twenty four buffer pictures (eight of each picture type) and four practice pictures. Materials for the arithmetic task were problems of addition and/or subtraction of two single digits. Pictures were presented centrally on a white computer screen with a resolution of $1024 \times 768$ pixels using E-Prime software.

\section{Procedure}

A $2 \times 3$ repeated measure design was used, with list composition (pure, mixed) as the between-subjects factor and picture type (negative, related neutral, random neutral) as the within-subjects factor. In both pure and mixed list conditions, participants studied three lists of 15 pictures each, presented in a counterbalanced order. In the pure list condition, participants studied one list with 15 negative pictures, one list with 15 related neutral pictures, and one list with 15 random neutral pictures. In the mixed list condition, all three picture types (five negative, five related neutral, and five random neutral) were included in each list. In this condition, the three picture types were pseudorandomized within list with the constraint that three pictures of the same type could not be presented consecutively. The order of presentation of the three lists in both pure and mixed list conditions was fully counterbalanced across participants.

Two buffer items were inserted before and after the experimental pictures in each list to control for primacy and recency effects. Buffer items were randomly allocated to each list, but in the pure list condition buffers always were of the same type as the experimental pictures (e.g. negative pictures served as buffers in the negative picture list). Study was intentional, with an orienting task of visual-complexity rating. To displace items from working memory, a 1-minute retention interval followed the list, during which participants performed an arithmetic judgment task, which engaged visual processing, decision making, and verbalization processes.

Memory for the pictures was then tested with free recall, following Bradley et al.'s (1992) procedure. Participants were asked to describe verbally the pictures they remembered. The recall instructions emphasized a succinct, yet informative, description (e.g. "a car accident", "a man sweeping"). The exact instructions for this task were as follows:

"Please recall all the pictures you can remember in any order. The experimenter will tape your recall. Try not to describe each picture in detail (1-4 words are usually enough). If the experimenter isn't sure which picture you have in mind, she will ask you about it at the end. The experimenter will tell you when time is up."

The experimenter coded the responses as matches or non-matches of study pictures, and asked participants to elaborate on any ambiguous descriptions at the end of the recall period to overcome potential confusions mentioned in Bradley et al. (1992). The elaboration probes, and the use of verbal recall, deviated from Bradley et al., but just like them, we found that in almost all cases, matching the descriptions to pictures was clear and straightforward. As noted below, inter-rater agreement was high.

Participants were tested individually. Before the experiment started, they practiced in all the tasks used in the study (visual complexity rating, arithmetic judgment, picture recall).

Each picture was presented for two seconds, followed by a four-second inter-stimulus interval, which was included to reduce carry-over effects of emotion. The interval was chosen on the basis of data from Talmi, Schimmack, Paterson, and Moscovitch, 2007, which showed that concurrent task performance is elevated when participants watch emotional pictures simultaneously, but returns to baseline after four seconds, suggesting that participants no longer allocate extra resources to emotional pictures at that time. During the inter-stimulus interval, participants rated the previous picture for visual complexity on a 1-3 scale which was presented at the bottom of an otherwise blank screen. The arithmetic task started immediately after the list. In this task, they participants were given a series of arithmetic problem-pairs, which they read out loud, and then indicated with a key-press which problem in the pair generated a larger answer. Participants were encouraged to solve correctly as many problems as they could.

Participants were then given three minutes to recall the pictures they remembered in any order by describing them verbally. The experimenter coded the responses as matches or non-matches of study pictures, and asked participants to elaborate on any ambiguous descriptions at the end of the recall period. After completing the experiment, participants were asked about strategies that they used during the task, and then were debriefed and thanked.

\section{Results}

As predicted, emotionally-enhanced memory was only found when either distinctiveness or relatedness were allowed to play a role, and was no longer significant when these factors were controlled (see Fig. 1). All the following analyses use a significance value of $p=.05$ and report the halfwidths of the $95 \%$ confidence interval (CI) for pairwise comparisons. 


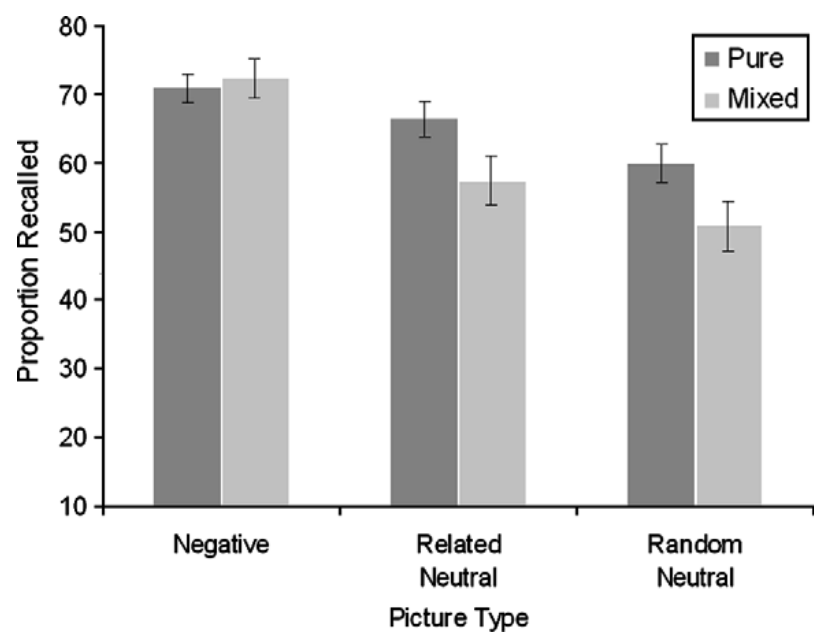

Fig. 1. Free recall in Experiment 1 as a function of list composition (pure or mixed) and picture type (emotional, related neutral, or random neutral). Error bars represent standard errors.

The experimenter transcribed all recall output. An independent rater scored $25 \%$ of the transcribed recall. Inter-rater agreement was high (Spearman-Brown boosted reliability $>.98)$. Here, and in the following experiments, we counted the number of pictures of each type - negative, related neutral and random neutralthat participants recalled from each one of the experimental lists. These scores were converted to proportions and submitted to a repeated-measures ANOVA with picture type as a within-subject factor and list composition (pure, mixed) varying between subjects. The effect of picture type was significant, $F(2,98)=23.40$, MSE $=140.93, p<.001$, partial $\eta^{2}=.32$. The main effect of list composition was marginally significant, as participants recalled more pictures in the pure list condition than in the mixed list condition, $F(1,49)=3.64$, $\mathrm{MSE}=105.12, p=.06$, partial $\eta^{2}=.07$. Critically, the interaction between list composition and picture type was significant, $\quad F(2,98)=3.276, \quad \mathrm{MSE}=140.93$, $p<.05$, partial $\eta^{2}=.06$. Bonferonni-corrected paired $t$-tests showed that in the mixed list condition, negative pictures $(M=72.38, \mathrm{SD}=13.87)$ were recalled better than random neutral pictures $(M=50.79, \mathrm{SD}=18.19)$, $t(20)=5.69, p<.001$, a difference of $21.69, \mathrm{CI}=7.90$. Negative pictures were also recalled better than related neutral pictures $(M=57.46, \mathrm{SD}=16.80), t(20)=5.27$, $p<.001$, Cohen's $d=.97$ (Cohen, 1988), a difference of 14.92, CI $=5.91$. There was a non-significant trend towards recalling more related neutral than random neutral pictures. In the pure list condition, similarly to the mixed list condition, negative pictures $(M=70.89, \mathrm{SD}=10.43)$ were recalled better than random neutral pictures $(M=60.00, \quad \mathrm{SD}=13.33)$, $t(29)=4.09, p<.001$, a difference of $10.89, \mathrm{CI}=5.45$. In contrast to the mixed list condition, however, in the pure list condition, the advantage of negative over related neutral pictures was not significant $(M=66.44$, $\mathrm{SD}=12.93), t(29)=1.31, p>.10$, Cohen's $d=.38$, a difference of $4.45, \mathrm{CI}=6.92$. The trend towards recalling more related than random neutral pictures was not significant after the Bonferonni correction was applied.

Independent sample $t$-test showed no difference between the recall of negative pictures in the two list composition conditions, $t<1$. Related and random neutral pictures were recalled significantly better in the purelist relative to the mixed-list condition [related: $t(49)=2.07, p<.05$; random: $t(49)=2.16, p<.05]$, but the difference was no longer significant after the Bonferonni correction was applied.

\section{Additional analyses}

Intrusions. Participants also recalled some items that did not match any experimental picture on the list (intrusions). The number of intrusions was relatively low and represented $2.7 \%$ of the total recall in the pure list condition, and $4.25 \%$ of the total recall in the mixed list condition.

Output order. To the extent that negative pictures have relatively stronger memory traces than neutral pictures, possibly due to the effects of distinctiveness on encoding processes, they should be recalled earlier than neutral items (Wixted, Ghadisha, \& Vera, 1997). Such a bias would result in output interference, which could reduce recall of non-emotional items (Roediger \& Schmidt, 1980). Note that an output bias is not eliminated by a distractor task and, therefore, it does not imply that participants were maintaining the negative items in working memory (Smith, 1971). In order to explore output bias effects, we counted the number of times each participant recalled a picture of each type in output positions 1-15. Score ranged from $0 \%$, if the participant did not recall any picture of a certain type in a specific output position, $33 \%$ if they recalled a picture of that type in that position once, $66 \%$ for two such recalls, and $100 \%$ if they recalled a picture of that type in that position in all three lists. We submitted these output frequency scores to a 15 (output position) $\times 3$ (picture type) repeated measures ANOVA (see Fig. 2a). As expected, we found significant main effects of type, $F(2,40)=19.19, \mathrm{MSE}=252.02$, $p<.001$, partial $\eta^{2}=.49$, and a significant main effect of output order, $F(14,280)=14.58, \quad \mathrm{MSE}=239.42$, $p<.001$, partial $\eta^{2}=.42$. More importantly, the interaction of type and output position was significant, $F(28,560)=2.66, \quad \mathrm{MSE}=670.49, \quad p<.001, \quad$ partial $\eta^{2}=.12$, documenting the bias to recall emotional items early within the recall output stream. We probed this bias further by dividing, for each participant, the output frequency scores by the participant's own probability of recalling items of each of the picture types, and submitted the corrected scores to the same ANOVA. The effect of 

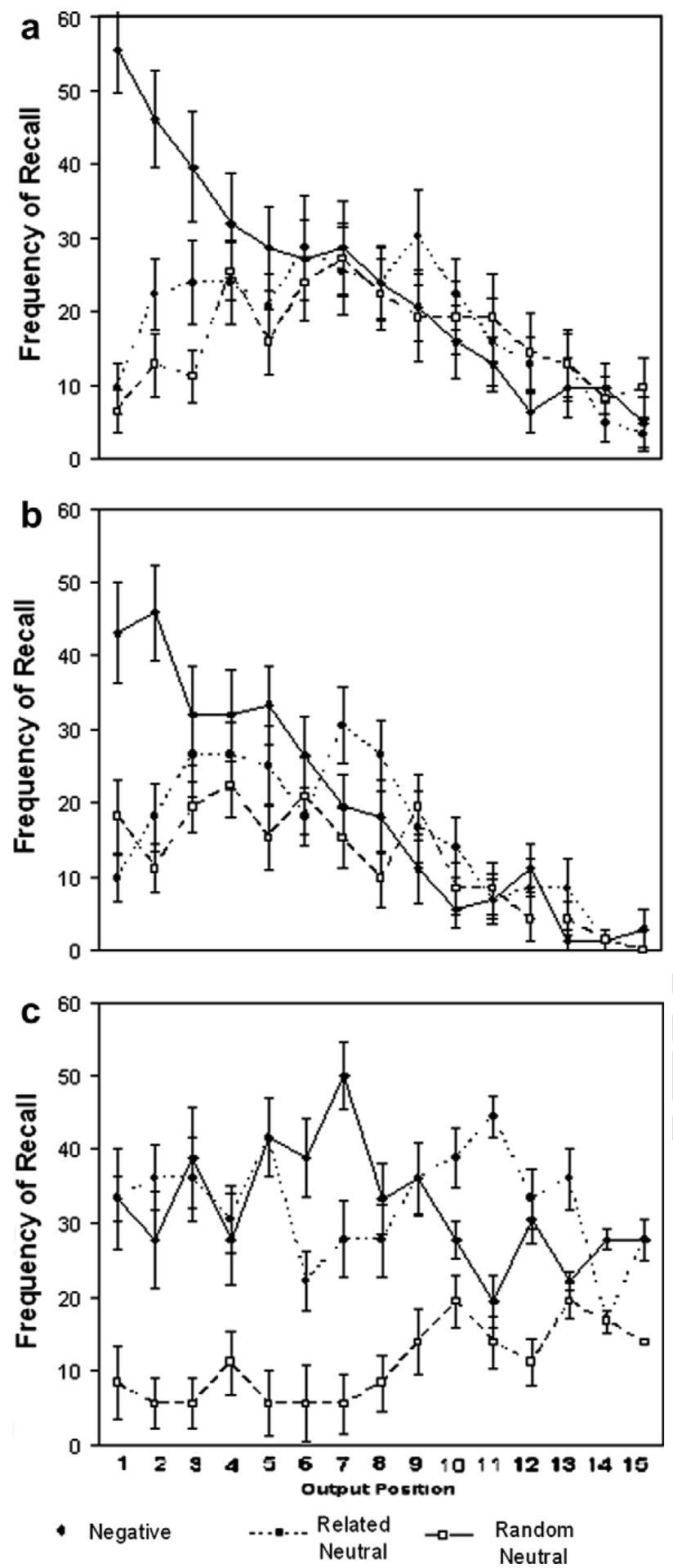

Fig. 2. Frequency of recall (in percentile) of each output position in the mixed list condition of Experiment 1 (a), 2B (b) and 3 (c), as a function of picture type (emotional, related neutral, or random neutral). Error bars represent standard errors.

output position and the interaction with type remained significant.

Orienting task. We analyzed the visual complexity ratings with a repeated measures ANOVA with picture type as a within subject factor and list composition as between subject factor. Although pilot rating data indicated that picture types were equivalent in visual complexity, picture type had a significant effect on participants' visual complexity rating at encoding, $F(2,96)=6.25$, MSE $=.11, p<.01$, partial $\eta^{2}=.11$. The main effect of list composition was not significant, but interestingly, in line with the memory data, participants differed in their visual complexity ratings of the same pictures as a function of list composition, $F(2,96)=3.05$, MSE $=.11, p=.05$, partial $\eta^{2}=.06$. In line with the memory data, the effect of picture type on complexity ratings was not significant for the pure lists, $p>.10$, but it was significant for the mixed list, $F(2,40)=6.33, \mathrm{MSE}=.16, p<.05$, partial $\eta^{2}=.24$. Bonferonni-corrected pairwise comparisons showed that negative pictures were rated as more complex than related neutral pictures, $p<.05$, with a non-significant trend in the same direction in the comparison of negative and random neutral pictures.

Distractor task. Performance on the distractor task did not differ according to list composition. T-tests showed that list composition did not significantly affect the number of problems attempted in the arithmetic judgment task, $p>.10$, or the accuracy in solving the problems, $p>.10$. Within the pure list condition, picture type did not significantly affect the number of problems attempted, $p>.10$ or the accuracy in solving them, $p>.10$.

\section{Discussion}

In the mixed list condition of Experiment 1, we found better memory for emotional over random-neutral items, replicating numerous previous studies (for review, see Labar \& Cabeza, 2006). Further, in this condition, the mnemonic advantage of emotional items was smaller but still substantial and significant relative to related neutral items. This finding allows us to conclude that emotional memory advantage in mixed lists is not only a result of their higher inter-relatedness. In the pure list condition, when both relatedness and relative distinctiveness were controlled (in the comparison of emotional and related neutral lists), emotional items no longer had a significant advantage, but their advantage reappeared when only distinctiveness was controlled (in the comparison of emotional and random-neutral lists). This pattern of findings is consistent with the hypothesis that the higher distinctiveness and relatedness of emotional items in combination are responsible for of the effect of emotion on memory.

While we failed to reject the null hypothesis that memory for emotional and related-neutral pictures was equivalent, there was a trend in that direction. Since the comparison between emotional and related neutral items in the pure-list condition is crucial for our 
argument, we must now consider the implications of the numerical memory advantage for emotional pictures in Experiment 1. In the mixed-list condition, the effect size of the comparison between emotional and related-neutral pictures $(d=.97)$ exceeded Cohen's (1988) proposed convention for large effect $(d=.80)$. Given a sample of 30 participants and $\alpha$-level of $5 \%$, the probability of rejecting the null hypothesis is .87 . While Experiment 1 , therefore, had sufficient power to detect an effect of a similar magnitude to the one found in the mixed-list condition, it did not have enough power to detect medium or small effects. Although the trend towards better memory for emotional pictures could reflect low power to detect a real difference in memory as a function of item type, we hypothesized that it stemmed more from participants' ability to utilize the inter-relatedness of the neutral list to support memory. Individual differences could allow some participants to be better at this than others. Experiment 2 was designed to overcome this possible confound by giving participants practice on recalling related-neutral items, so as to increase their awareness of how the items were related.

We now consider the possibility that the trend towards recalling more emotional items than related items in the mixed-list condition, but not in the pure-list condition, occurred not because we eliminated the relative-distinctiveness advantage of emotional items in the latter condition, but because presenting the emotional pictures one after the other made them less arousing. In other words, pure-list presentation could have habituated participants so that they were no longer aroused by the negative pictures presented at late serial positions in the list.

Note that in order for this explanation to account for our findings, it is necessary to assume that emotional arousal is the factor that drives immediate memory enhancement. This contradicts previous findings in animals, which suggested that the effects of emotional arousal on memory are only present when the study-test interval is prolonged, namely, when memory is tested hours or even days, but not seconds or minutes, after the initial exposure to the emotional event (Bianchin, Mello-e-Souza, Medina, \& Izquierdo, 1999). The dependency of the effect on the time of test is explained by the fact that while emotional arousal activates the amygdala during original exposure, the amygdala only influences memory by improving the long-term consolidation of emotional memory traces (McGaugh, 2004).

There are additional considerations that cast doubt on the habituation explanation for the absence of emotionally-enhanced memory. Firstly, data on skin conductance responses to emotional words show that although emotional items are less arousing in a pure list presentation than in a mixed list presentation, they are still more arousing than neutral items even in pure lists (e.g. Walker \& Tarte, 1963; Smith, Bradley, \& Lang,
2005). Importantly, the effect of novelty on skin conductance response seems equivalent for personally significant and neutral stimuli (Ben Shakhar, 1994), so that habituation should conserve the relatively stronger responses to emotional stimuli. This smaller, but significant, difference in arousal in favor of emotional pictures, which was presumably also present in the purelist condition of our experiment, should have still resulted in significant memory enhancement, but it did not. Secondly, physiological measures of emotional arousal, such as heart rate, freezing, and startle-reflex magnitude are sustained and even increase after exposure to a series of unpleasant pictures (Azevedo et al., 2005; Smith et al., 2005; Bradley, Cuthbert, \& Lang, 1996). Finally, and most importantly, even if one assumes arousal can drive immediate memory enhancement, then if arousal is reduced in the pure list condition, memory for emotional pictures should have been better in the mixed list than in the pure list condition. To the contrary, memory for emotional items in Experiment 1 was equivalent in both list-composition conditions. What changed was memory for the neutral pictures which was worse in the mixed-list condition.

While the pure-list condition served to control differences in relative distinctiveness, it also served to control for the effect of biased output order, which was demonstrated here when participants recalled emotional items first in the mixed list condition. This underlying cause for the output bias may be the result of the higher relative strength of emotional items (Wixted et al., 1997), but its presence even after controlling for overall memory recall suggests that it may also be due to a strategic decision to report the emotional items first. Therefore, the replication of emotional memory benefit over random neutral ones in the pure-list condition, where output bias cannot operate, demonstrates that the classic effect seen with mixed-list presentation is not the result of output bias.

Participants in the mixed list condition rated emotional pictures as more visually complex than neutral pictures. This effect is interesting because pilot participants, as well as participants in the pure-list condition, rated the picture types as equally complex. This finding could be due to the higher relative distinctiveness of emotional pictures in a mixed list, but its absence in Experiments $2 \mathrm{~B}$ and 3 suggests it was probably due to chance.

In our earlier study with words (Talmi \& Moscovitch, 2004), memory was not enhanced for emotionally-arousing words in either pure or mixed lists. Because our previous and current studies obtained similar pure-list results, the discrepancy between the mixedlist results may be due to the higher distinctiveness of emotional pictures relative to emotional words, when embedded in neutral items, rather than to differences in arousal between the two material types. Further 
research is required to decide whether the difference in distinctiveness, like the difference in arousal, is related to goal-relevance, as we have previously proposed.

\section{Experiment 2A}

The non-significant trends in Experiment 1 towards better memory for emotional over related-neutral pictures, and related over random-neutral pictures, suggested that some participants might (i) not realize that the neutral items belong to an ad-hoc category, or (ii) need some experience with the memory task, before they are able to utilize item inter-relatedness to encode and retrieve pictorial stimuli optimally. Failure of participants to take advantage of item-relatedness in the neutral set could mask the effects of relatedness which we are investigating.

Experiment 2A, therefore, replicated the pure-list condition of Experiment 1 but added an extensive practice stage, during which participants saw three additional lists of pictures. If emotional memory really is better than neutral memory, above and beyond the effects of emotion on relatedness and distinctiveness, it should be apparent here in the comparison of emotional and related-neutral items. Otherwise, the addition of a practice phase should bring memory levels for these two item types even closer together than they were in Experiment 1. We also expected to find a significant memory advantage for related over random neutral items, which was only present as a trend in Experiment 1. Note that the additional practice was intended to decrease the difference between memory for related and emotional pictures and increase the difference between related and random neutral pictures. To check that the practice phase was working as planned, we tested participants' awareness of the thematic structure of the related neutral lists by having them write down the themes of each studied list.

\section{Method}

\section{Participants}

Twenty-four undergraduate students from the University of Toronto ( 10 males, mean age $22, \mathrm{SD}=2.4)$ participated in the study for course credit or a pay of $\$ 10$.

\section{Material}

Experimental materials were identical to the materials used in the pure list condition of Experiment 1, but included another, similarly constructed picture set for generalization purposes (see Table 1, sets 1 and 2). Participants were randomly allocated to picture set conditions. We analyzed the pictures in the two sets with a series of 2 (picture set) $\times 3$ (picture type) univariate
ANOVA analyses. There was no significant main effect of picture set or interactions between set and picture type, $p>.10$. Picture types statistically differed in valence, $F(2,84)=211.85, p<.001$, and in emotional arousal, $F(2,84)=220.09, p<.001$. Planned contrasts showed that the negative pictures were significantly more negatively valenced, $p<.001$, and more arousing, $p<.001$, relative to both types of neutral pictures. The two neutral types did not differ in valence, $p>.10$, or in arousal, $p>.10$. Picture types also differed in semantic relatedness, $F(2,84)=92.29, p<.001$, with lower scores for random neutral pictures, $p<.001$, but no difference between the related neutral categorized and negative pictures, $p>.10$. Picture types did not differ on people presence, visual complexity, or brightness, all $p$ values $>.10$.

To corroborate our claims that absolute distinctiveness was higher for emotional pictures than for neutral pictures, four graduate students and research assistants rated how unusual/novel each picture was. The instructions emphasized that the rating is not relative, but corresponds to the frequency of similar images in one's life. There was high agreement among raters, standardized Cronbach $\alpha>$.90. The effect of type was significant, $F(2,6)=96.12, p<.001$, and it did not interact with set; the main effect of set was also not significant, $p=.06$. Planned contrasts showed that the emotional pictures were rated as more unusual/novel than the neutral pictures, $p<.01$, but the two neutral types did not differ from one another, $p>.10$.

In addition, in Experiment $2 \mathrm{~A}$ we added three similarly constructed practice lists, which included lists of novel negative, related neutral (professions) and random neutral pictures.

\section{Procedure}

The procedure was almost identical to the procedure used in the pure list condition of Experiment 1. The main changes were the use of group testing (2-5 participants per group), which required written rather than verbal output during free recall and silent performance of the distractor. For participants, there was no distinction between the practice and the experimental lists. Participants were given a short break halfway through the experiment, before the beginning of the experimental stage. The order of presentation of the three lists was fully counterbalanced across subjects, but all participants were given the same list order in the practice and the experimental phases. At the end of the experiment, participants were told that each list had a theme, and were asked to write down their guess as to what each theme was.

\section{Results}

As shown in Fig. 3, emotional items were recalled significantly better than random-neutral items, replicating 


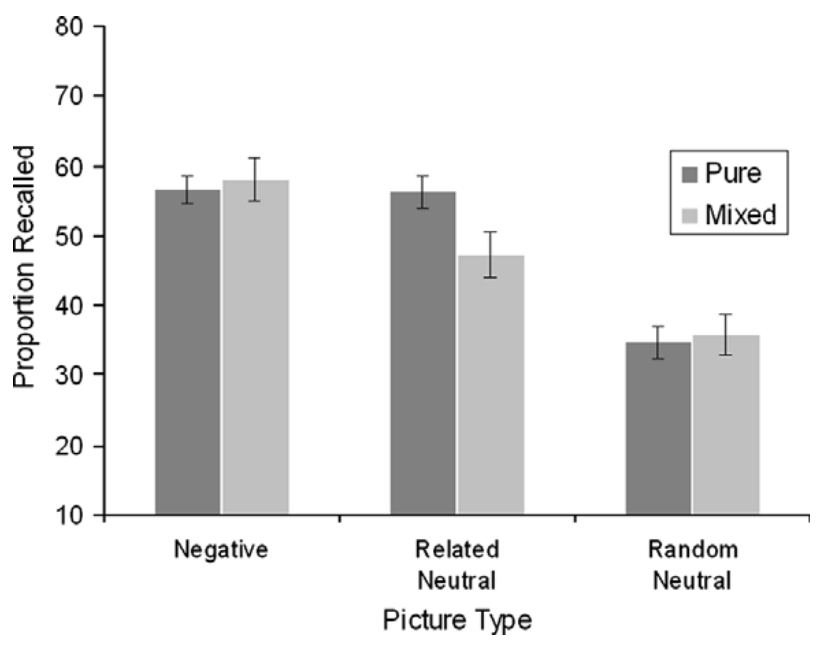

Fig. 3. Free recall in Experiment 2A (pure lists) and 2B (mixed lists) as a function of picture type (emotional, related neutral, or random neutral). Error bars represent standard errors.

the classic emotionally-enhanced memory effect, but there was only a minimal difference between memory for emotional and related-neutral items. Related-neutral items were recalled better than random-neutral items. The effect of picture type was significant, $F(2,44)=31.31, \quad \mathrm{MSE}=121.47, \quad p<.001, \quad$ partial $\eta^{2}=.59$. Planned contrasts shows that emotional pictures $(M=56.67, \mathrm{SD}=10.95)$ were recalled better than random neutral pictures $(M=34.72, \quad \mathrm{SD}=13.89)$, $F(1,22)=47.00, \quad \mathrm{MSE}=245.95, \quad p<.001, \quad$ partial $\eta^{2}=.68$, a difference of $21.95, \mathrm{CI}=6.56$, but not significantly better than related neutral pictures $(M=56.38$, $\mathrm{SD}=15.47), F(1,22)=.01, \mathrm{MSE}=247.69, p>.9$, partial $\eta^{2}<.01$, a difference of $0.29, \mathrm{CI}=6.50$. Paired $t$-test showed that related-neutral items were recalled better than random-neutral ones, $t(23)=6.9, p<.001$, a difference of 21.66, CI $=6.49$. The effect size of the comparison between emotional and related-neutral pictures was smaller than Cohen's (1988) suggested convention for a small effect size and we consider it negligible. Neither the main effect of picture set, nor the interaction with this factor was significant, $p>.10$.

\section{Additional analyses}

Intrusions. The number of intrusions was relatively low and represented $2.44 \%$ of the total recall (an average of .12 emotional, .08 related-neutral and .12 random-neutral intrusions).

Description length. An alternative explanation for emotionally-enhanced memory in free recall is that it is easier to describe the emotional pictures. This alternative can be tested by examining the number of words used in recalling each picture. The effect of picture type on the word count was significant, $F(2,46)=13.60$, $\mathrm{MSE}=.85, p<.001$, partial $\eta^{2}=.37$. Contrary to the suggestion that describing emotional pictures is quicker, participants in fact used more words to describe emotional than related neutral pictures, $p<.001$, or random neutral pictures, $p<.001$, which did not differ from one another, $p>.9$. The fact that participants used the same number of words to describe both related and random neutral pictures, while memory performance markedly differed between these pictures types, suggests that description length is not a critical factor in memory for pictures.

Orienting task. Visual complexity ratings did not differ according to picture type, $p>.01$, or picture set, $p>.10$.

Distractor task. Picture type and picture set did not significantly affect the number of problems attempted in the arithmetic judgment task, $p>.1$, nor the accuracy in solving the problems, $p>.10$.

Knowledge of themes. Participants' written reports of themes were scored leniently, so that participants were classified as 'aware' of the emotional or related-neutral them if they mentioned a plausible unifying theme for these pictures (e.g. 'house' or 'family' would be classified as 'aware' for the domestic-activity category). During practice, $46 \%$ of participants did not report what the theme of the related-neutral pictures was, but only $17 \%$ did not report the theme of the experimental related-neutral pictures. While this could result from forgetting the practice pictures at that point of the experiment, it is in line with our suggestion that practice helped participants note the relatedness information and take advantage of it. All participants reported the negative theme of the emotional pictures, which repeated at practice and at test.

Relationship between relatedness and free recall. To validate our measure of relatedness, we counted the number of times each picture was recalled to arrive at a picturerecall score. There was a significant correlation between the relatedness of an individual picture to other pictures in the set and the number of times this picture was recalled, $r=.35, p<.05$.

\section{Discussion}

Experiment 2A replicated the pure-list results of Experiment 1. Because we controlled differences in the way participants approached the recall task by giving them extensive practice with the same materials and procedures, the present results were clearer than those of Experiment 1. These data show that memory for emotional and related-neutral items is not significantly different when relative distinctiveness and relatedness cannot aid memory for emotional material. The comparison of related and random-neutral items showed that organization 
significantly improved memory for neutral items. The better memory for emotional over randomly-selected neutral items replicates previous findings, but the inclusion of the related-neutral condition suggests that this effect is driven by organization, not emotion.

\section{Experiment 2B}

Since the extension of the practice phase had a strong impact on performance in Experiment 2A, we designed Experiment $2 \mathrm{~B}$ to ensure that the effects we obtained in the mixed list condition in Experiment 1 would also be replicated with the procedures used in Experiment $2 \mathrm{~A}$.

\section{Method}

\section{Participants}

Twenty-four undergraduate students from the University of Toronto (10 males, mean age 21.54, $\mathrm{SD}=2.21$ ) participated in the study for a pay of $\$ 10$.

\section{Materials and procedure}

We used picture set 1 (used in Experiment 1, see Table 2) and the practice lists from Experiment 2A. The procedure was identical to the procedure used in Experiment $2 \mathrm{~A}$.

\section{Results}

As predicted, emotional items were recalled better than related neutral items, which were recalled better than random neutral items (see Fig. 3). The effect of picture type was significant, $F(2,46)=30.08$, $\mathrm{MSE}=98.52, p<.001$, partial $\eta^{2}=.57$. Planned contrasts showed that emotional pictures $(M=58.05$, $\mathrm{SD}=15.66)$ were recalled better than related neutral pictures $\quad(M=47.22, \quad \mathrm{SD}=15.47), \quad F(1,23)=12.39$, $\mathrm{MSE}=227.30, p<.01$, partial $\eta^{2}=.35$, a difference of $10.83, \mathrm{Ci}=6.36$. Related neutral pictures were recalled better than random neutral pictures $(M=35.83$, $\mathrm{SD}=14.15), F(1,23)=20.40, \mathrm{MSE}=152.58, p<.001$, partial $\eta^{2}=.47$, a difference of $11.39, \mathrm{CI}=5.21$.

\section{Additional analyses}

Intrusions. The number of intrusions was relatively low and represented $3.54 \%$ of the total recall. (an average of .37 emotional, .12 related-neutral, and .25 random-neutral intrusions).

Description length. The effect of picture type on the word count was significant, $F(2,46)=11.94$, $\mathrm{MSE}=.79, p<.001$, partial $\eta^{2}=.34$. Contrary to the idea that description of emotional pictures was easier and faster, participants used more words to describe emotional than related neutral, $p<.01$, or random neutral pictures, $p<.001$, which did not differ from one another, $p>9$.

Output order. Again, a significant main effects of type was found, $F(2,46)=31.48, \mathrm{MSE}=160.89, p<.001$, partial $\eta^{2}=.58$, and a significant main effect of output order, $F(14,322)=29.99$, MSE $=214.74, p<.001$, partial $\eta^{2}=.57$. More importantly, the interaction of type and output position was significant, $F(28,644)=2.47$, MSE $=544.53, p<.001$, partial $\eta^{2}=.10$, documenting the bias to recall emotional items early within the recall output stream (see Fig. 2b). Again, the effect of output position and the interaction with type remained significant after correcting these scores for levels of recall of each picture type.

Orienting task. Visual complexity ratings did not differ according to picture type, $p>0.1$.

Distractor task. Picture set did not significantly affect the number of problems attempted in the arithmetic judgment task, $p>.1$, or the accuracy in solving the problems, $p>.1$.

Knowledge of themes. While during practice $71 \%$ of participants did not report the theme of the related-neutral pictures, only $46 \%$ did not report the theme of the experimental related-neutral pictures, again underscoring the importance of the practice phase. As in Experiment $2 \mathrm{~A}$, all participants reported the negative theme of the emotional pictures. In order to keep list-length constant, participants here were exposed to fewer related-neutral items in mixed lists (5) than in the pure lists (15). Consequently, it may have been more difficult to notice the related-neutral theme which may help account for the higher percentage of unaware participants in Experiment 2B relative to Experiment 2A. Importantly, the advantage of emotional over related neutral pictures was the same regardless of theme report [aware: $t(12)=2.49, \quad p<.05$; unaware: $t(10)=2.37$, $p<.05]$.

Relationship between organization and free recall. There was a significant correlation between the relatedness of an individual picture to other pictures in the set and the number of times this picture was recalled, $r=.33, p<.05$ (comparable to $r=.35$ in Experiment 2A).

Analysis of experiments $2 \mathrm{~A}$ and $2 \mathrm{~B}$. Results from Experiments $2 \mathrm{~A}$ and $2 \mathrm{~B}$ were analyzed together with Experiment as a between-subject factor. Experiment 2 replicated the significant interaction between picture type and list type found in Experiment 1, $F(2,92)=3.98$, MSE $=134.77, p<.05$, partial $\eta^{2}=.08$. 
The main effect of Experiment was not significant, but the main effect of picture type was, $F(2,46)=58.15$, $\mathrm{MSE}=108.90, p<.001$, partial $\eta^{2}=.56$. Importantly, there was no significant difference between the emotional lists, $t<1$, or the random-neutral lists, $t<1$, but the related-neutral lists were recalled better in the pure list than in the mixed-list condition, $t(46)=2.05, p<.05$, $.17<18.15$. In the mixed-list condition, the effect size of the comparison between emotional and related-neutral pictures $(f=.73)$ exceeded Cohen's (1988) proposed convention for large effect $(f=.40)$. If a large effect size was present in the population for pure lists, then given a sample of 24 participants and an $\alpha$-level of $5 \%$, the probability of rejecting the null hypothesis is greater than .78; if an effect size as large as that present in the mixed list comparison was present in the population for pure lists the probability of rejecting the null hypothesis rises to .98 .

\section{Discussion}

The results of Experiment 2B replicated the findings from the mixed list condition in Experiment 1. Memory was best for emotional items, and better than memory for equally-related neutral items. This result rules out the possibility that the additional practice that participants received in Experiment 2A, particularly the exposure to negative pictures, reduced the arousal they experienced when they saw the negative experimental pictures. The fact that relatedness significantly improved memory for neutral pictures in experiments $2 \mathrm{~A}$ and $2 \mathrm{~B}$, an effect which was only present as a trend in Experiment 1, supports our suggestion that the extensive practice allowed more participants to use relatedness to aid their memory.

One question the data from Experiment 2A and 2B raises is why all participants reported the emotional theme but not all reported the related-neutral theme. The reason could be due to our use of a different related-neutral theme for practice and experimental lists, whereas we repeated the negative-emotional theme in both phases. To anticipate, in Experiment 4 we show that even when both emotional and related-neutral themes are repeated, participants still reported the latter less frequently.

Data from Experiments 1 and 2 shows that emotionally-enhanced memory can be obtained whenever mixedlist stimulus presentation is used, even when item inter-relatedness is controlled, and participants are given practice to support utilization of relatedness, and also are aware of the theme of the related-neutral pictures. This suggests that relatedness does not account fully for emotionally-enhanced memory-differential distinctiveness also plays a role. The next experiment was designed to determine whether distinctiveness operates at encoding or retrieval.

\section{Experiment 3}

The higher distinctiveness of emotional items may improve memory because it evokes special encoding processes (McDaniel, Dornburg, \& Guynn, 2005; Schmidt, 1991). For instance, distinct items could attract attention, and enhanced attentional allocation to unusual items at encoding has been used to explain why they are remembered better (Jenkins \& Postman, 1948). Similarly, the higher distinctiveness of emotional items could lead to increased processing of the encoding context (e.g. MacKay et al., 2004), which has also been linked to improved memory (Hintzman \& Stern, 1978). Alternatively, the distinctiveness-based mnemonic advantage of emotional items may be solely based on retrieval processes (McDaniel et al., 2005; Schmidt, 1991). Unique features, unshared with non-emotional items, could make emotional items incongruent with the conceptual framework participants use at retrieval. The unique features would make the memory strength of emotional items better equipped to compete for retrieval, as Neath (1993), following Murdock (1960), has shown in his mathematical model of retrieval. The encoding and retrieval effects can work together to enhance memory of emotional items in mixed lists. In Experiment 3, we examined whether relative distinctiveness at retrieval was sufficient for the manifestation of an emotionally-enhanced memory effect.

When participants study a pure list and recall it immediately, emotional items do not have a relativedistinctiveness advantage either at encoding or retrieval. When they study a mixed list and recall it immediately, emotional items have a relative-distinctiveness advantage at both stages. To remove the effect of relative-distinctiveness only at the encoding stage, in Experiment 3 participants studied pure lists of emotional and neutral items, but study was not followed by immediate recall. The effect of relative distinctiveness was introduced at retrieval by giving participants a surprise final-recall test in which they recalled items from all three lists in any order. This paradigm allows emotional items to stand out relative to the neutral items only at retrieval. McDaniel et al. (2005) used a similar procedure to disentangle encoding and retrieval components of the bizarreness effect (better memory for bizarre sentences). Because the bizarreness effect was still present with this procedure, the authors concluded that relative distinctiveness at retrieval was sufficient for its manifestation. In Experiment 3 we investigated whether their finding also applies to emotional stimuli. Experiments 1 and 2 showed that purelist study leads to equivalent memory for emotional and related-neutral items. If an emotional memory advantage emerges with the final-recall procedure, it then can be attributed to the effects of relative distinctiveness at retrieval. 
Because memory strength is correlated with the amount of processing items received at encoding (Wixted et al., 1997), eliminating special encoding processes towards emotional items by blocking item type (McDaniel et al., 2005) should also make all items equally "strong", and reduce the bias to recall emotional items first in this experiment.

\section{Method}

\section{Participants}

Thirty-six undergraduate students from the University of Toronto (seven males, mean age 21.19, $\mathrm{SD}=1.92)$ participated in the study for a pay of $\$ 10$. An additional eight participants reported that they expected a final free recall test and were removed.

\section{Material and procedure}

We used the two picture sets as in Experiment 2A. The procedure was almost identical to the one used in Experiment 2A, with the following differences. Participants were told in advance that only some of the lists will be tested, but that they had to study each one as if it will be followed by a memory test. Final recall was never mentioned. In fact, all three practice lists were tested just as in Experiment 2, but none of the experimental lists was tested. After the last experimental list, participants were engaged in a final distractor task for five minutes, a visuospatial mental rotation pencil-andpaper task. Following that, they were asked to recall all the pictures they had not recalled earlier, and were also told all of these items were studied in the second half of the experiment (after the break). The duration of the final recall test was 9 min.

\section{Results}

The results replicated those of Experiment 2A: at final recall, there was no significant memory advantage for emotional pictures relative to related-neutral pictures (see Fig. 4). The effect of picture type was significant, $F(2,70)=35.31, \quad$ MSE $=195.81, \quad p<.001, \quad$ partial $\eta^{2}=.51$. Planned contrasts showed that emotional pictures were not recalled significantly better than related neutral ones $(M=46.66, \mathrm{SD}=18.17), F(1,34)=2.54$, $\mathrm{MSE}=353.62, p>.10$, partial $\eta^{2}=.07$, a difference of $5, \mathrm{CI}=6.27$. Related-neutral pictures were recalled better than random neutral pictures, $t(35)=5.46, p<.001$, a difference of 21.11, $\mathrm{CI}=7.84$. Replicating previous studies the results again showed that when emotional memory and relatedness are confounded, emotional memory enhancement is found in the comparison of emotional and random-neutral pictures $(M=25.55, \mathrm{SD}=15.16)$, $F(1,34)=90.25, \quad$ MSE $=271.19 \quad p<.001, \quad$ partial $\eta^{2}=.73$, a difference of $26.11, \mathrm{CI}=5.56$. Neither the main effect of set nor the interaction was significant, $p>.10$.

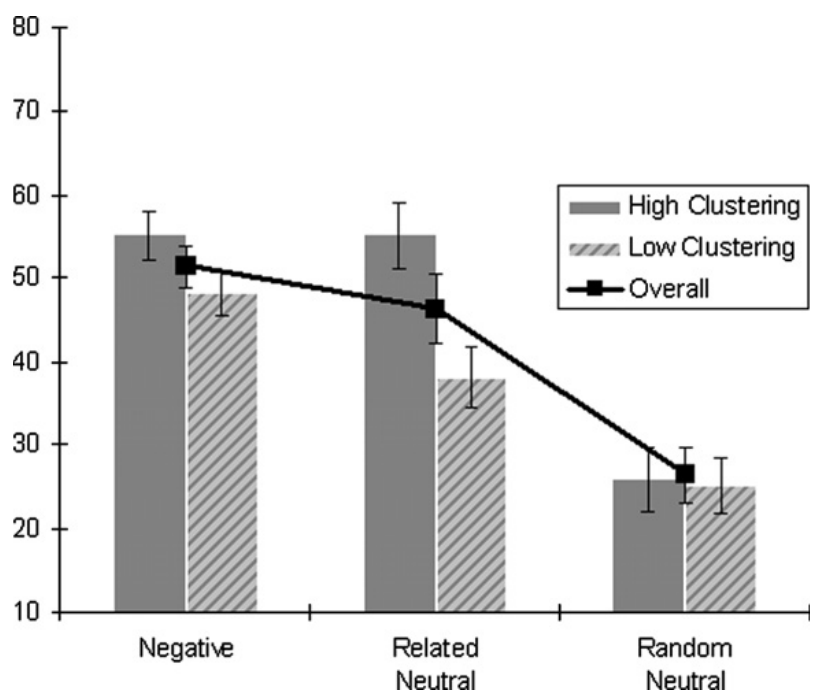

Fig. 4. Free recall in Experiment 3 as a function of picture type (emotional, related neutral, or random neutral) and participants' semantic clustering score (high or low). Error bars represent standard errors.

\section{Additional analyses}

Intrusions. The final recall procedure led to an increase in the number of intrusions, which represented $9 \%$ of the total recall (an average of .58 emotional, .53 related-neutral and .42 random-neutral intrusions). Because of the slightly higher number of intrusions, we classified them according to type. Picture type did not have a significant effect on the number of intrusions, $p>.10$.

Description length. The effect of picture type on the word count was significant, $F(2,64)=15.30$, MSE $=.99, p<.001$, partial $\eta^{2}=.33$. Participants used more words to describe emotional than both types of neutral pictures, $p<.001$, which did not differ from one another, $p>.9$.

Orienting task. Visual complexity ratings did not differ according to picture type, $F<1$.

Distractor task performance. Picture type did not significantly affect the number of problems attempted in the arithmetic judgment task, $F<1$. Whereas for the accuracy in solving the problems, picture type had a significant effect, $F(2,70)=3.91$, MSE $=.002, p<.05$. Post hoc tests showed that participants were less accurate after viewing the negative pictures $(M=86 \%$, $\mathrm{SD}=17 \%)$ relative to the related-neutral $(M=89 \%$, $\mathrm{SD}=15 \%$ ), but this difference was not significant after Bonferonni correction was applied.

Trend towards better recall of emotional over relatedneutral pictures. We explored the free recall data to understand this trend better, and observed that some participants tended to recall items from the same list 
consecutively, and then switch to another list. We quantified this tendency with the list-based semantic clustering index (Stricker, Brown, Wixted, Baldo, \& Delis, 2002), a per-list score of the tendency to report items of the same type consecutively, which controls for differences in the number of items recalled. Within the context of the present experiment, a high clustering score means that participants tended to focus on a single list at a time, rendering the final recall procedure similar to immediate pure-list recall, and thereby masking the potential effect of relative distinctiveness at retrieval. In line with this possibility, a median-split analysis of participants with higher and lower semantic clustering index showed that only participants who did not cluster their recall very much exhibited emotional memory advantage (see Fig. 4). When we included clustering score as a factor in the ANOVA we found that picture type still had a significant effect, $F(2,68)=38.50$, MSE $=179.62, p<.001$, partial $\eta^{2}=.53$, but it was qualified by a significant interaction with clustering, $F(2,68)=3.38, p<.05$, partial $\eta^{2}=.09$. Bonferonnicorrected paired $t$-tests showed that participants whose clustering score was higher did not recall emotional pictures significantly better than related-neutral ones, $t<0.01$, and the previously present trend in this direction was no longer present in this half of the sample. By contrast, participants with a lower clustering score recalled emotional pictures better than related-neutral ones, $t(17)=2.76, \quad p=.01, \quad$ a difference of 10 , $\mathrm{CI}=7.65$. Participants with lower clustering scores also had overall worse memory, $F(1,34)=6.60$, $\mathrm{MSE}=279.76, p<.05$, partial $\eta^{2}=.16$. Bonferonnicorrected $t$-tests showed that the drop in memory performance between participants with high- and low-clustering scores was significant for related-neutral pictures $t(34)=3.15, p<.01$, a difference of 17.04, $\mathrm{CI}=10.98$. The same trend for negative pictures was no longer present after the Bonferonni correction was applied, $t(34)=1.78, \quad p=.08$, a difference of 7.04 , $\mathrm{CI}=8.0$. Both participants groups had equivalent memory for random neutral pictures, $t<1$.

Output order. We did not observe an output bias in this experiment, even after correcting the scores as in Experiment $1, p>.1$, see Fig. 2 (panel C). Output order did not interact with the semantic clustering scores, $p>.10$.

\section{Discussion}

In Experiment 3, emotional items were not relativelydistinct at encoding, because all items were studied in pure lists. Although the final recall procedure was employed in order to examine the effects of relative distinctiveness at retrieval, individual differences in the tendency to organize recall by study-list reduced the effectiveness of this procedure. Participants with high clustering scores tended to recall each study list separately, and their memory data were similar to those of participants who recalled pure lists immediately (Experiment 2A). By comparing these participants with those with low-clustering scores, we determined that the strategy of recalling related-neutral items together improved memory for them, so that emotional items no longer had a significant memory advantage. Organized memory strategy thus proved to be an important predictor of emotional memory advantage even when item interrelatedness was controlled.

By contrast, participants with low clustering scores recalled items from all lists simultaneously, instead of clustering their output by study list. They showed an emotional memory advantage, and their data resembled data from Experiment 2B, in which both study and retrieval contexts were mixed. The straightforward interpretation of this finding is that relative distinctiveness at retrieval is sufficient for the emotional memory advantage to become manifest. However, because organized recall is an individual-difference variable, it is also possible that participants with low clustering scores were overall less prone to use organizational strategies to aid performance. The emotional memory advantage these participants exhibited could be due either to the utilization of relative distinctiveness, which pushed memory for emotional items up, or to poor organization, which pushed memory for related-neutral items down. The data supports the organization interpretation because low clusterers displayed a trend for poorer memory for emotional pictures, relative to high clusterers. This interpretation is further supported by the finding that memory for related-neutral items was significantly poorer in the low-clusterers, with no reduction in their memory for random-neutral items; utilization of the relative distinctiveness of emotional items should not have influenced memory for relatedneutral items more than memory for random-neutral ones. The comparison of Experiments 1 and 2, as well as the comparison of Experiments $2 \mathrm{~A}$ and $2 \mathrm{~B}$, highlighted the importance of organizational strategies for remembering related-neutral items. Findings from Experiment 3 converge on the same idea. Although the memory pattern of the two clustering groups was easily interpreted within the organization framework, it is important to note that the analysis of clustering scores was conducted post hoc so that other differences between the two groups could provide an alternative explanation for the differential memory pattern they exhibited.

Experiment 3 replicated the finding from previous experiments of a smaller advantage of emotional over related-neutral items, relative to their larger advantage when compared with unrelated neutral items, this time using a final-recall procedure. Experiment 3 also showed that even when participants retrieve emotional and 
neutral items together, emotional memory advantage is not dependent on a bias to recall the emotional items first.

One open question about the data from Experiments 1 and 2 is why participants found it easier to notice the negative than the neutral theme, as reflected in their theme report. Although the "negative" theme was arguably as much ad hoc as the "domestic" or the "profession" theme of the neutral items, participants clearly had a harder time identifying the latter. With the setup we used in Experiments 2 and 3, participants possibly could have found it easier to notice the negative theme because they were exposed to two lists of negative pictures (at practice and during the experimental trials), but experienced each neutral theme only once. The repetition of the emotional, but not the related-neutral theme could have given emotional memory an unfair advantage. In Experiment 4 we repeated both the related-neutral and the emotional theme.

\section{Experiment 4}

In Experiment 4, we equated the frequency of participants' encounter with related-neutral and negative themes by using the same domestic-scene category during both practice and experimental phases. If the less-frequent report of the related-neutral theme was dependent on this procedural detail, participants should now report it as frequently as the emotional theme. We predicted, however, that the less-frequent report of the related-neutral theme reflects the more volitional and effortful nature of neutral-item encoding, and predicted that even when theme exposure was equated, participants would report the related-neutral theme less frequently than the emotional theme. This study also aimed to replicate the critical finding of equivalent memory for emotional and related-neutral pictures, this time with equal exposure to both themes.

\section{Method}

\section{Participants}

Twenty-four undergraduate students from the University of Toronto participated in the study for course credit and were randomly allocated to the full or the divided-attention condition (18 females, mean age $18.73, \mathrm{SD}=1.91)$.

\section{Materials}

We used the same set 1 of experimental pictures (see Table 1). Practice pictures consisted of the same emotional and random-neutral pictures used in Experiment 2 , but the related-neutral 'profession'-themed pictures were replaced by domestic scenes, the same theme used in the experimental related-neutral list.

\section{Procedure}

The procedure was almost identical to that used in Experiment 2A, with slight changes because of the inclusion of other conditions which are not of central interest here. The differences were the addition of an auditory task between the practice and the experimental phases (not analyzed here) and the elimination of the orienting task during picture encoding.

\section{Results}

The difference between memory for negative and related neutral pictures was minimal, but memory for random neutral pictures was worse. The effect of picture type was significant, $F(2,92)=16.58, \mathrm{MSE}=160.20$, $p<.001$, partial $\eta^{2}=.42$. Planned contrasts showed that emotional pictures $(\mathrm{M}=61.66, \quad \mathrm{SD}=13.79)$ were recalled slightly, but not significantly, worse than related-neutral pictures $(M=66.94, \quad \mathrm{SD}=16.74)$, $F(1,23)=2.84, p>.10$, partial $\eta^{2}=.11$, a difference of $5.38, \mathrm{CI}=6.57$, but better than random-neutral pictures $(M=46.67, \quad \mathrm{SD}=19.26), \quad F(1,23)=17.86, \quad p<.001$, partial $\eta^{2}=.44$, a difference of $14.99, \mathrm{CI}=7.57$. From these comparisons it is clear that related-neutral pictures were recalled better than random-neutral pictures. We again failed to reject the null hypothesis that memory for emotional pictures is better than memory for neutral ones, but this time, memory for neutral pictures was numerically higher.

\section{Additional analyses}

Intrusions. The number of intrusions was relatively low and represented $1.72 \%$ of the total recall (an average of .21 emotional, .04 related-neutral and .08 random-neutral intrusions).

Distractor task. Picture type did not significantly affect the number of problems attempted in the arithmetic judgment task, $p>.1$, or the accuracy in solving the problems, $p>.10$.

Knowledge of themes. All participants reported the negative theme of the emotional pictures, but $37.5 \%$ of participants did not report the related-neutral theme. Theme report did not correlate with free recall, $p>.10$. Neither 'aware' nor 'unaware' participants had better memory for emotional pictures relative to related-neutral ones, $p>.10$.

\section{Discussion}

Experiment 4 replicated the crucial 'negative' finding obtained in the pure-list condition of Experiment 1 and Experiments 2A: when study blocked item type, the emotional memory advantage in the comparison of emotional and related-neutral items was no longer 
present. Experiment 4 replicated this effect when the related-neutral theme repeated at the practice and test phases, demonstrating that it could not be attributed to the repetition of the emotional theme, but not the neutral one, in our previous experiments. The repetition of the related-neutral theme in both practice and test phases in Experiment 4 likely gave those items a slight boost over related-neutral items in Experiment 2A, and allowed them to be remembered marginally better than emotional items.

Because this alteration did not eliminate the lower frequency of neutral theme report, we reasoned that the negative theme may simply be recognized more easily relative to any ad-hoc neutral category. This obtains because evaluation is considered a primary way of meaning-making (Osgood, Suci, \& Tannenbaum, 1957); the evaluative dimension may also be recognized quickly and automatically (Pratto \& John, 1991). In other words, participants may not have needed inferences to know they disliked some of the stimuli they saw, but they did need some inferences to realize what the theme of neutral pictures was (Zajonc, 1980). Notably, despite the difference in noting the theme of the emotional and the related-neutral items, memory for the two types was not significantly different, perhaps because participants relied more on inter-item associations than on a general conceptualization of the list's theme when they recalled the pictures.

\section{Combined analysis of the critical contrast in Experiments $1-4$}

In the pure-list study conditions of all experiments presented above, we failed to reject the null hypothesis that memory for emotional pictures is better than memory for related-neutral ones when item type is blocked at study. The effect size obtained in these comparisons was in the small-to-medium range according to Cohen's convention (Experiment 2A: $f<.0001$; Experiment 3, pure list, all participants: $f=.27$; Experiment 3, pure list, high-clusterers: $f<.0001$ ), apart from Experiment 4 where a large effect was obtained but in the opposite direction $(f=.35)$, namely, an advantage for relatedneutral items. By contrast, the effect sizes obtained in the mixed list condition were larger than Cohen's 'large' convention (Experiment 1: Cohen's $d=.97$; Experiment 2B: Cohen's $f=.73$ ). We can therefore safely conclude that blocking item type significantly reduces the magnitude of the emotional memory advantage.

Here we aggregated data from all pure-list conditions (The pure-list condition in Experiment 1, and Experiments 2A, 3 and 4), a total of 114 participants, in order to increase our power to detect a difference between emotional and related-neutral pictures. We analyzed the effect of picture type (emotional, related neutral) with Experiment as a between-subject factor. The main effect of Experiment was significant, $F(3,110)=19.04$,
MSE $=248.85, p<.001$, partial $\eta^{2}=.34$, but importantly, the effect of Type was not significant, $F<1$, partial $\eta^{2}=.004$, nor did it interact with Experiment, $F(3,110)=2.04, \quad \mathrm{MSE}=149.65, \quad p>.10, \quad$ partial $\eta^{2}=.05$. Given this aggregated sample of 114 participants and an $\alpha$-level of .05 , the probability of rejecting the null hypothesis is greater than 0.85 if an effect size of $f=.20$, smaller than Cohen's 'medium' convention of $f=.25$, is present in the population (Cohen, 1988). We conclude, therefore, that if memory for emotional pictures is better than memory for equally-related neutral pictures, this represents only a small effect, which is unlikely to be detected with typical sample sizes.

\section{General discussion}

The experiments reported here consistently demonstrated that controlling the differences in relatedness and relative distinctiveness between emotional and neutral items eliminated the significant immediate memory advantage emotional items had over neutral ones when those factors were not controlled. The analysis across all four experiments ascertained that the critical null effect in the comparison between pure lists of emotional and related-neutral items is of negligible magnitude, and even with considerable statistical power, non-significant.

This conclusion is supported by the finding that unlike the negligible difference between memory for pure lists of emotional and related-neutral items, memory for emotional items was significantly better than memory for neutral ones as long as either organization or distinctiveness was not controlled. In mixed lists, the higher relative distinctiveness of emotional items allowed them to be recalled better than related neutral ones. In addition, we replicated the classic memory advantage of emotional over random-neutral items in both pure and mixed lists conditions. We attribute this effect to organizational factors in pure lists, and to organizational and distinctiveness factors in mixed lists.

Experiment 3 was designed to allow relative distinctiveness to operate at retrieval but not at encoding by asking for a final free recall of pictures presented in pure lists at encoding. While Experiment 3 again showed that overall, emotional pictures were not recalled significantly better than related-neutral ones in a final recall test, there was a trend in that direction, which was accounted for by participants' tendency to recall items from each study list separately. Participants who clustered their recall to a greater degree recalled related-neutral items just as well as emotional ones. Their data provided a replication for the critical finding from the pure-list conditions in the other three experiments reported here. By contrast, participants who did not cluster their recall by study list remembered emotional items better than related-neutral ones. We suggested 
that the reason for this was their poor organization of the related-neutral items, rather than the relative distinctiveness of emotional items, but further research would be required to support this interpretation.

Notably, throughout this series of experiments, we have seen that organization is critical to bring memory for neutral material to the level of memory of emotional material. When organization is controlled in principle, but not in practice, e.g. because of individual differences in a surprise final-recall test, or as a result of the amount of practice participants have with free-recall tasks (compare pure-list memory in Experiments 1 and 2), memory for related-neutral pictures will remain relatively low, and emotional memory advantage would be evident.

The absolute distinctiveness advantage of emotional items did not translate to better memory in the pure list conditions, but it made emotional items relatively distinct, which in turn improved memory for them in the mixed list condition. This pattern is similar to the finding that memory for a clothed model in a series of nudes is better than memory for the nudes because the clothed model is relatively distinct, despite the higher absolute distinctiveness (and arousal) of the nudes (Schmidt, 2002), and is in agreement with Schmidt's (1991) conclusion that relative distinctiveness influences memory more than absolute distinctiveness.

One caveat that was inherent to the free recall procedure is that we only have information about memory for the gist of the picture which is sufficient for identification, but not about memory for all the details in the pictures which the participant may remember. Although in the pure-list condition there was no difference in memory for the gist of emotional and related-neutral pictures, participants may have had better memory for details in the emotional pictures, which would not be revealed in our procedure. Further research should equate pictures for the number of ideas or objects they contain and test memory with a more fine-grained paradigm, such as requiring participants to recall everything they can remember about the picture, with cuing, if necessary. That said, the benefit of emotional over neutral items is typically in memory for the gist of the picture, with worse memory for the details of emotional pictures (Adolphs, Tranel, \& Buchanan, 2005; see Christianson, 1992; for review).

How does relative distinctiveness at encoding enhance memory for emotional items? An intriguing aspect of the comparison between the pure and mixed-list conditions in Experiments 1 and 2 is that emotional memory was equivalent in both list composition conditions, but memory for neutral pictures was lower in the mixed-list condition. This finding is closely in line with the experimentally-induced retrograde amnesia effect: poor memory for neutral items presented just before emotional items (e.g. Strange, Hurlemann, \& Dolan, 2003; Tulving, 1969). While the cause for the retrograde amnesia effect has yet to be determined, it was shown to be dependent on the emotionally-arousing nature of the stimulus (Schulz, 1971; Schmidt, 2002), which may require intact amygdala and noradrenergic function in order to exert its effect (Strange et al., 2003). Retrograde-amnesia effects are dependent on the length of the inter-stimulus interval between the emotional event and the preceding neutral event: it is obtained with short intervals (e.g. Detterman \& Ellis, 1972; Schulz, 1971; Tulving, 1969), but not long ones (Anderson, Wais, \& Gabrieli, 2006), and may even be reversed with intermediate intervals (Anderson et al., 2006). The reduced memory for the item following a distinct one in the list is typically attributed to the continued processing of the salient item during subsequent item presentation, and thus is more strongly linked with the effect of distinctiveness than with the non-cognitive aspects of arousal. Future research will be needed to determine whether retrograde and anterograde amnesia plays a role in a mixed-list presentation even when participants are given ample time to process each of the items, as was the case here.

\section{Theoretical implications}

Emotionally-arousing items in typical experimental settings and in many life situations outside the laboratory, are also more related, more distinct, and better attended at encoding. Relatedness, distinctiveness, and attentional resources support memory in both immediate and delayed tests. According to the most parsimonious interpretation, the present results support the suggestion that the effect of emotion on memory is completely mediated by its effect on organization and distinctiveness.

There is much evidence, however, that, in addition to this cognitively-mediated process, a unique neurobiological and automatic process exists that enhances long-term consolidation of emotionally-arousing items (see McGaugh, 2004; for review). This process represents a direct influence of emotional arousal on memory and results in an even stronger effect of emotion on memory when memory is tested after a relatively prolonged delay (hours and days) relative to when it is tested shortly (seconds or minutes) after study (e.g. Hamann et al., 1999; LaBar \& Phelps, 1998; Sharot \& Phelps, 2004). In this vein, the modal finding in earlier research with words is that delayed benefits of emotion are more stable than immediate ones, which are obtained often, but not always (for review see Craik \& Blankstein, 1975; Eysenck, 1976). Our working hypothesis is that both mediation and modulation mechanisms play a role in delayed emotionally-enhanced memory effects, as shown in the combined mediation/modulation model (see Fig. 5). 


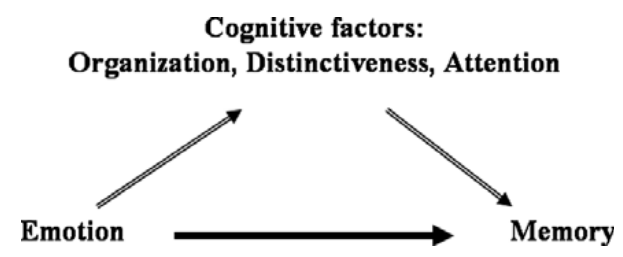

Fig. 5. The combined mediation-modulation model of emotionally-enhanced memory. The open-lined arrows represent the cognitive mediation mechanism. The arrow from emotion to relatedness, distinctiveness and attention represents the suggestion that emotional items are more related, distinct and attended than neutral items. The arrow from those cognitive factors to memory represent the suggestion that these cognitive attributes of emotional items is the reason for their typical mnemonic advantage, at least in immediate testing. The boldlined arrow represents the direct path from emotion to memory postulated by the modulation hypothesis.

A different theoretical perspective on the present data set is that organization and distinctiveness are more important for emotional memory advantage than they are for emotional memory per se. That is, organization and distinctiveness benefit neutral items, and raise memory for them to the level of emotional items, but the possibility remains that emotional items rely on such cognitive factors less; instead, they exert at least part of their effect by influencing memory directly even in immediate tests. In the present study we have seen that by rigorously controlling neutral items, to make them more similar to emotional ones, we brought memory for neutral material up to the level of emotional memory. By contrast, neither the manipulation of list composition, nor individual strategies of organization had a substantial effect on emotional memory: it always stayed just as high. It may well be possible to reduce emotional memory by using more extreme measures, but the important point is that memory for emotional items is more resilient than memory for neutral items. Therefore, it is possible that even when all the cognitive consequences of emotional arousal are eliminated, and memory for emotional items is equivalent to memory for related-neutral ones, the underlying mechanisms are not the same.

We recently tested these alternatives by focusing on attention as a mediating factor in emotional memory (Talmi et al., 2007). The central finding was that dividing attention at encoding reduced memory for related-neutral pictures, but not memory for negative emotional pictures (that study used mixed lists, but we have since replicated it with pure lists, Talmi, 2006; for similar findings, see Bush \& Geer, 2001; Kensinger \& Corkin, 2004; Kern, Libkuman, Otani, \& Holmes, 2005). The effect of attention was, therefore, similar to the effect of distinctiveness in the experiments reported here. Another aspect of the Talmi et al. (2007) data was that negative emotional pictures captured attention at encoding, so that their processing was 'less divided' than the processing of neutral pictures. The overall pattern could be interpreted according to the mediation model: the enhanced attention for emotional items could account for their subsequent better recall. Further investigation, however, showed that the attentionally-mediated account of the emotional memory advantage was wrong. A mediator analysis of the contrast of negative and neutral pictures demonstrated that the effect of emotion via attention was not significant. Instead, emotion had a direct effect on memory that was significant even when attentional differences were accounted for statistically or controlled empirically. The possibility remains that organization and distinctiveness operate like attention in the Talmi et al. study, and may be more important for neutral memory than for emotional memory, a topic we are currently pursuing. If the more arousing an item is, the more it activates non-cognitive processes such as those proposed by McGaugh (2004), then one prediction that follows from this interpretation is that more arousing emotional material than was used in the present study might be remembered better than neutral material even in pure lists.

In conclusion, equating cognitive processes such as relatedness, distinctiveness, and attention between emotional and neutral items significantly reduces or eliminates the emotionally-enhanced memory effect, suggesting that emotional items may exert their effects on memory indirectly, mediated by these cognitive factors. Other studies, however, suggest that emotional items affect memory directly by influencing physiological processes acting on memory structures and modulating their activity. By this view, equating for all the mediating factors simply enhances memory for neutral items to the level observed for emotional items, which may rely on direct, modulated effects rather than indirect, mediated ones. Clearly, these two interpretations are not mutually exclusive, but at the moment, it is not possible to determine their relative importance to immediate emotional memory advantage. What is clear from our studies is that the true effect of emotion on memory cannot be evaluated properly without equating for these cognitive factors. The direction we hope future research will take is to determine how mediated and modulated effects of emotion on memory interact with one another both immediately and at long delays.

\section{Acknowledgment}

This work was supported by NSERC Grant \# CFC 205055 Fund 454119 to Morris Moscovitch. The authors thank Joseph J. Williams for his assistance with scoring, Marty Niewiadomski, for his feedback on the analysis of Experiment 3, and Elisa Ciaramelli and Nachshon Meiran for reading a previous draft of this 
manuscript, and Marilyne Ziegler for excellent technical support.

\section{References}

Anderson, A. K., Wais, P. E., \& Gabrieli, J. D. (2006). Emotion enhances remembrance of neutral events past. Proceedings of the National Academy of Sciences of the United States of America, 103(5), 1599-1604.

Adolphs, R., Denburg, N. L., \& Tranel, D. (2001). The amygdala's role in long-term declarative memory for gist and detail. Behavioral Neuroscience, 115, 983-992.

Adolphs, R., Tranel, D., \& Buchanan, T. W. (2005). Amygdala damage impairs emotional memory for gist but not details of complex stimuli. Nature Neuroscience, $8(4), 512-518$.

Azevedo, T. M., Volchan, E., Imbriba, L. A., Rodrigues, E. C., Oliveira, J. M., Oliveira, L. M., et al. (2005). A freezing-like posture to pictures of mutilation. Psychophysiology, 42, 255-260.

Ben Shakhar, G. (1994). The roles of stimulus novelty and significance in determining the electrodermal orienting response: interactive versus additive approaches. Psychophysiology, 31(4), 402-411.

Bianchin, M., Mello-e-Souza, T., Medina, J. H., \& Izquierdo, I. (1999). The amygdala is involved in the modulation of longterm memory but not in working or short-term memory. Neurobiology of Learning and Memory, 71, 127-131.

Blake, T. M., Varnhagen, C. K., \& Parent, M. B. (2001). Emotionally arousing pictures increase blood glucose levels and enhance recall. Neurobiology of Learning and Memory, $75,262-273$.

Bradley, M. M. (1994). Emotional memory: a dimensional analysis. In S. Van Groot, N. E. Van de Poll, \& J. Sargeant (Eds.), The emotions: Essays on emotion theory (pp. 97-134). Hillsdale, NJ: Erlbaum.

Bradley, M. M., \& Lang, P. J. (1994). Measuring emotion: the self-assessment manikin and the semantic differential. Journal of Behavior Therapy and Experimental Psychiatry, 25, 49-59.

Bradley, M. M., Greenwald, M. K., Petry, M. C., \& Lang, P. J. (1992). Remembering pictures: pleasure and arousal in memory. Journal of Experimental Psychology: Learning, Memory and Cognition, 18, 379-390.

Bradley, M. M., Cuthbert, B. N., \& Lang, P. J. (1996). Picture media and emotion: effects of a sustained affective context. Psychophysiology, 33(6), 662-670.

Buchanan, T. W., Etzel, J. A., Adolphs, R., \& Tranel, D. (2006). The influence of autonomic arousal and semantic relatedness on memory for emotional words. International Journal of Psychology, 61(1), 26-33.

Bush, S. I., \& Geer, J. H. (2001). Implicit and explicit memory of neutral, negative emotional, and sexual information. Archives of Sexual Behavior, 30, 615-631.

Christianson, SA. (1992). Emotional stress and eyewitness memory: a critical review. Psychological Bulletin, 112(2), 284-309.

Cohen, N. (1988). Statistical power analysis for the behavioral sciences (2nd ed.). Hillsdale, NJ: Lawrence Earlbaum Associates.
Craik, F. I. M., \& Blankstein, K. R. (1975). Psychophysiology and human memory. In P. H. Venables \& M. J. Christie (Eds.), Research in psychophysiology (pp. 388-417). London: Wiley.

Detterman, D. K., \& Ellis, N. R. (1972). Determinants of induced amnesia in short-term memory. Journal of Experimental Psychology, 95(2), 308-316.

Dewhurst, A. A., \& Parry, L. A. (2000). Emotionality, distinctiveness, and recollective experience. European Journal of Cognitive Psychology, 12, 541-551.

Dolan, R. J. (2002). Emotion, cognition, and behavior. Science, 298, 1191-1194.

Einstein, G. O., \& McDaniel, M. A. (1987). Distinctiveness and the mnemonic benefits of bizarre imagery. In M. A. McDaniel \& M. Pressley (Eds.), Imagery and related mnemonic processes: theories, individual differences and applications (pp. 78-102). New York: Springer-Verlag.

Eysenck, M. W. (1976). Arousal, learning, and memory. Psychological Bulletin, 83, 389-404.

Gardiner, J. M., Craik, F. I., \& Birtwistle, J. (1972). Retrieval cues and release from proactive inhibition. Journal of Verbal Learning and Verbal Behavior, 11, 778-783.

Hamann, S. B., Ely, T. D., Grafton, S. T., \& Kilts, C. D. (1999). Amygdala activity related to enhanced memory for pleasant and aversive stimuli. Nature Neuroscience, 2, 289-293.

Hamann, S. B. (2001). Cognitive and neural mechanisms of emotional memory. Trends in Cognitive Sciences, 5, 394400.

Hamann, S. B., Cahill, L., McGaugh, J. L., \& Squire, L. R. (1997). Intact enhancement of declarative memory for emotional material in amnesia. Learning and Memory, 4(3), 301-309.

Hintzman, D. L., \& Stern, L. D. (1978). Contextual variability and memory for frequency. Journal of Experimental Psychology: Human Learning and Memory, 4, 539-549.

Hunt, R. R., \& Elliot, J. M. (1980). The role of nonsemantic information in memory: Orthographic distinctiveness effects on retention. Journal of Experimental Psychology: General, 109, 49-74.

Hunt, R. R., \& McDaniel, M. A. (1993). The enigma of organization and distinctiveness. Journal of Memory and Language, 32, 421-445.

Jenkins, W. O., \& Postman, L. (1948). Isolation and 'spread of effect' in serial learning. American Journal of Psychology, 61, 214-221.

Kensinger, E. A., \& Corkin, S. (2004). Two routes to emotional memory: distinct neural processes for valence and arousal. Proceedings of the National Academy of Sciences of the United States of America, 101(9), 3310-3315.

Kensinger, E. A., Brierley, B., Medford, N., Growdon, J. H., \& Corkin, S. (2002). Effects of normal aging and Alzheimer's disease on emotional memory. Emotion, 2, 118-134.

Kern, R. P., Libkuman, T. M., Otani, H., \& Holmes, K. (2005). Emotional stimuli, divided attention, and memory. Emotion, 5(4), 408-417.

Labar, K. S., \& Cabeza, R. (2006). Cognitive neuroscience of emotional memory. Nature Reviews: Neuroscience, 7(1), $54-64$.

LaBar, K. S., \& Phelps, E. A. (1998). Arousal-mediated memory consolidations: role of the medial temporal lobe in humans. Psychological Science, 9, 490-493. 
Lang, P.J., Bradley, M.M., \& Cuthbert, B.N. (1999). International affective picture system (IAPS): Instruction manual and affective ratings. Technical Report A-4.

Lazarus, R. S. (1991). Emotion and adaptaion. New York, NY: Oxford University Press.

MacKay, D. G., Shafto, M., Taylor, J. K., Marian, D. E., Abrams, L., \& Dyer, J. R. (2004). Relations between emotion, memory and attention: evidence from taboo stroop, lexical decision, and immediate memory tasks. Memory \& Cognition, 32(3), 474- 487.

Mandler, G. (1967). Organization and memory (Vol. 1). Oxford, England: Academic Press.

Mandler, G. (1980). Recognizing: the judgment of previous occurrence. Psychological Review, 87, 252-271.

Maratos, E. J., \& Rugg, M. D. (2001). Electrophysiological correlates of the retrieval of emotional and non-emotional context. Journal of Cognitive Neuroscience, 13, 877-891.

McDaniel, M. A., Dornburg, C. C., \& Guynn, M. J. (2005). Disentangling encoding versus retrieval explanations of the bizarreness effect: implications for distinctiveness. Memory \& Cognition, 33, 270-279.

McGaugh, J. L. (2004). The amygdala modulates the consolidation of memories of emotionally arousing experiences. Annual Reviews Neuroscience, 27, 1-28.

McRae, K., \& Boisvert, S. (1998). Automatic semantic similarity priming. Journal of Experimental Psychology: Learning, Memory, \& Cognition, 24(3), 558-572.

Murdock, B. B. Jr., (1960). The distinctiveness of stimuli. Psychological Review, 67, 16-61.

Neath, I. (1993). Contextual and distinctiveness processes and the serial position function. Journal of memory and Language, $32,820-840$.

Ochsner, K. N. (2000). Are affective event strictly recollected or simply familiar? The experience and process of recognizing feelings past. Journal of experimental psychology: General, 129, 242-261.

Osgood, C. E., Suci, G. J., \& Tannenbaum, P. H. (1957). The measurement of meaning. Urbana, IL: University of Illinois Press.

Palomba, D., Angrilli, A., \& Mini, A. (1997). Visual evoked potentials, heart rate responses and memory to emotional pictorial stimuli. International Journal of Psychophysiology, 27, 55-67.

Phelps, E. A., LaBar, K. S., \& Spencer, D. D. (1997). Memory for emotional words following unilateral temporal lobectomy. Brain and Cognition, 35, 85-109.

Phelps, E. A., LaBar, K. S., Anderson, A. K., O'Connor, K. J., Fulbright, R. K., \& Spencer, D. D. (1998). Specifying the contributions of the human amygdale to emotional memory: a case study. Neurocase, 4, 527-540.

Pratto, F., \& John, O. P. (1991). Automatic vigilance: the attention-grabbing power of negative social information. Journal of Personality and Social Psychology, 61, 380-391.

Roediger, H. L., \& Schmidt, S. R. (1980). Output interference in the recall of categorized and paired-associate lists. Journal of Experimental Psychology: Human Learning and Memory, 6, 91-105.

Schmidt, S. R. (1984). Encoding and retrieval processes in the memory for conceptually distinctive events. Journal of
Experimental Psychology: Learning, Memory, and Cognition, 11(3), 565-578.

Schmidt, S. R. (1994). Effects of humor on sentence memory. Journal of Experimental Psychology: Learning, Memory, and Cognition, 20(4), 953-967.

Schmidt, S. R. (2002). Outstanding memories: the positive and negative effects of nudes on memory. Journal of Experimental Psychology: Learning, Memory, and Cognition, 28, 353-361.

Schmidt, S. R. (1991). Can we have a distinctive theory of memory? Memory \& Cognition, 19, 523-542.

Schulz, L. S. (1971). Effects of high-priority events on recall and recognition of other events. Journal of Verbal Learning and Verbal Behavior, 10(3), 322-330.

Sharot, T., \& Phelps, E. A. (2004). How arousal modulates memory: disentangling the effects of attention and retention. Cognitive, Affective and Behavioral Neuroscience, 4, 294-306.

Smith, A. D. (1971). Output interference and organized recall from long-term memory. Journal of Verbal Learning and Verbal Behavior, 10, 400-408.

Smith, J. C., Bradley, J. C., \& Lang, P. J. (2005). State anxiety and affective physiology: effects of sustained exposure to affective pictures. Biological Psychology, 69(3), 247-260.

Strange, B. A., Hurlemann, R., \& Dolan, R. J. (2003). An emotion-induced retrograde amnesia in humans is amygdala- and beta-adrenergic-dependent. Proceedings of the National Academy of Sciences of the United States of America, 100(23), 13626-13631.

Stricker, J. L., Brown, G. G., Wixted, J., Baldo, J. V., \& Delis, D. C. (2002). New semantic and serial clustering indices for the California Verbal Learning Test-second edition: background, rationale, and formulae. Journal of the International Neuropsychological Society, 8(3), 425-435.

Symons, C. S., \& Johnson, B. T. (1997). The self-reference effect in memory: a meta-analysis. Psychological Bulletin, 121(3), 371-394.

Talmi, D. (2006). The role of attention and organization in emotional memory enhancement. Unpublished doctoral dissertation, University of Toronto.

Talmi, D., \& Moscovitch, M. (2004). Can semantic relatedness explain the enhancement of memory for emotional words? Memory \& Cognition, 32, 742-751.

Talmi, D., Schimmack, U., Paterson, T., \& Moscovitch, M. (2007). The role of attention in emotional memory enhancement. Emotion, 7(1), 89-102.

Tulving, E. (1969). Retrograde amnesia in free recall. Science, 164(3875), 88-90.

Walker, E. L., \& Tarte, R. D. (1963). Memory storage as a function of arousal and time with homogeneous and heterogeneous lists. Journal of Verbal Learning and Verbal Behavior, 2, 113-119.

Wixted, J. T., Ghadisha, H., \& Vera, R. (1997). Recall latency following pure- and mixed-strength lists: a direct test of the relative strength model of free recall. Journal of Experimental Psychology: Learning, Memory, and Cognition, 23, 523-538.

Zajonc, R. B. (1980). Feeling and thinking: preferences need no inferences. American Psychologist, 35, 151-175.

Zald, D. H. (2003). human amygdala and the emotional evaluation of sensory stimuli. Brain Research Reviews, 41, 88-123. 